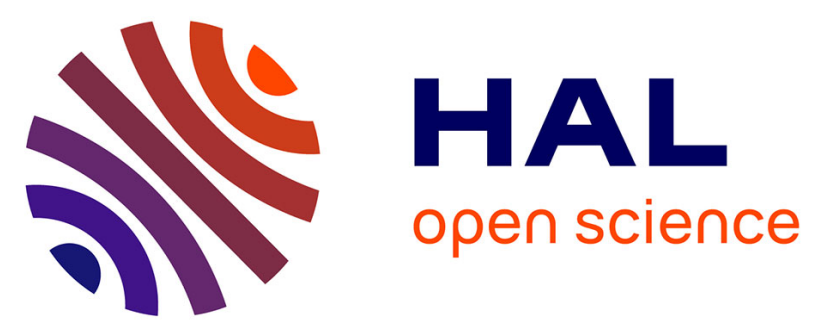

\title{
Short-time OD matrix estimation for a complex junction using Fuzzy-Timed High-Level Petri Nets
}

Krystyna Biletska, Marie-Hélène Masson, Sophie Midenet, Thierry Denoeux

\section{To cite this version:}

Krystyna Biletska, Marie-Hélène Masson, Sophie Midenet, Thierry Denoeux. Short-time OD matrix estimation for a complex junction using Fuzzy-Timed High-Level Petri Nets. 12th International IEEE Conference on Intelligent Transportation Systems, Oct 2009, St. Louis, United States. pp.1-6. hal00443746

\section{HAL Id: hal-00443746 \\ https://hal.science/hal-00443746}

Submitted on 4 Jan 2010

HAL is a multi-disciplinary open access archive for the deposit and dissemination of scientific research documents, whether they are published or not. The documents may come from teaching and research institutions in France or abroad, or from public or private research centers.
L'archive ouverte pluridisciplinaire HAL, est destinée au dépôt et à la diffusion de documents scientifiques de niveau recherche, publiés ou non, émanant des établissements d'enseignement et de recherche français ou étrangers, des laboratoires publics ou privés. 


\title{
Short-time OD matrix estimation for a complex junction using Fuzzy-Timed High-Level Petri Nets
}

\author{
Krystyna Biletska $^{* \dagger \S}$, Marie-Hélène Masson ${ }^{\dagger \ddagger}$, Sophie Midenet ${ }^{\S}$ and Thierry Denœux* ${ }^{* \dagger}$ \\ * Université de Technologie de Compiègne \\ $\dagger$ UMR CNRS 6599 Heudiasyc, BP 20529, 60205 Compiègne cedex, France \\ $\ddagger$ Université de Picardie Jules Verne, IUT de l'Oise \\ §Université Paris-Est, Institut National de Recherche sur les Transports et leur Sécurité (INRETS), Laboratoire GRETIA \\ 2 rue de la Butte Verte, 93166 Noisy le Grand cedex, France, E-mail: krystyna.biletska@utc.fr (corresponding author)
}

\begin{abstract}
The OD matrix at a signalised junction estimated for traffic light cycle represents a crucial information for transportation systems. A new dynamic two-steps method is proposed to estimate such an OD matrix. First, a vehicle conservation law is build in a dynamical way for each traffic light cycle using FuzzyTimed High-Level Petri Nets (FTHN). It is represented by an under-determinate system of equations whose unknowns are the elements of the OD matrix. Second, a fuzzy linear programming approach is used to solve this system. Since the data used to estimate the matrix are imprecise, they are represented as fuzzy numbers. Experimental tests made with real data provided by video cameras installed on a junction show the benefits of using an FTHN tool for this problem.
\end{abstract}

Index Terms-Fuzzy-Timed High-Level Petri Nets, fuzzy sets, origin-destination matrix estimation, signalised junction.

\section{INTRODUCTION}

The origin-destination (OD) matrix, that describes traffic demand at a junction, is a key element of most transport systems. Each element $b_{i j}$ of OD matrix $\mathbf{B}$ is a proportion of the flow of vehicles that come from entrance (origin) $i$ and go to exit (destination) $j$. Such a proportion is called the $O D$ flow rate. An OD matrix is generally deduced from vehicle counts made on each entrance and each exit of the junction during a given time interval. These counts are usually provided by magnetic loops embedded in the road surfaces and sensitive to metallic masses. The estimation can be obtained from a conservation law of vehicles which is a set of relationships between exit and entrance flow counts. In general, when loops are installed on every entrance and exit of the junction, the estimation problem is under-determined. Thus a solution is not unique and additional information such as a prior OD matrix is used to choose the OD matrix which corresponds best to the actual matrix. Some of the existing methods are based on the information minimisation principle [1], on maximisation of likelihood [2] or on Bayesian inference [3]. Other methods propose a recursive estimation of the OD matrix [4], [5]. Furthermore, the estimation problem can be represented as a constraint optimisation problem [6], [7].

The cited methods are well suited for the OD matrix estimation per quite large period of time $(\approx 15$ minutes). Since the changes in traffic demand can be more frequent, the estimation period of the OD matrix has to be as short as possible. In particular, the period should be equal to a traffic light cycle (duration of green-amber-red sequence) when we deal with a signalised junction. At INRETS/GRETIA we use such a shorttime estimation of the OD matrix as part of a diagnostic system for signalised junctions [8]. This system compares the impacts of different traffic control strategies expressed in terms of $\mathrm{CO}_{2}$ and pollutant emissions. One of the estimation methods which use traffic lights is described in [9], [10].

This paper considers the problem of reconstituting the origins and destinations of vehicle flows crossing a signalised junction, at each traffic light cycle. OD flow volumes are estimated using traffic lights and traffic measurements from video cameras installed at the junction. These measurements, provided every second, are the vehicle counts made on each entrance and exit of the junction and the number of vehicles stopped at each inner section of the junction. The data is subject to redundancy, imprecision and uncertainty. Such imperfections are linked to the reliability of video sensors, measurement conditions, traffic characteristics and drivers' behaviour. None of the cited methods takes into account the lack of precision of vehicle counts, the possible physical complexity of the junction and the traffic lights at the same time. Moreover they cannot be applied to the problem because the period of estimation is quite short.

The aim of this paper is to obtain the best estimates of the OD matrix by modelling the data imperfection, using a two-stage method. First, a conservation law of vehicles, which is represented by an inconsistent and under-determined system of equations, is built by Fuzzy-Timed High-Level Petri Nets (FTHN) at each traffic light cycle. Second, the fuzzy linear programming approach is proposed to overcome the inconsistency of the system and to model the data imperfection.

In [11] we proposed a similar two-stage method that solves the given problem by taking into account the inherent data imprecision. However, this method, based on the use of HighLevel Petri Nets (HLPN), does not consider the temporal imprecision related to the modelling of OD flows crossing the junction.

Numerical tests of two methods has been done using the 
data collected in a real experimental signalised junction fitted out with video cameras.

The rest of this paper is organised as follows. Section II describes the experimental site and traffic data collected on it, and states the problem. Section III introduces the model of segmentation of traffic flows crossing the signalised junction. Estimation method and experimental results are presented in Section IV. The last section concludes the paper.

\section{Problem Statement}

In this paper we address the problem of OD matrix estimation for a signalised junction with a complex structure. The experimental site, used for this purpose, is an isolated signalised junction of two double-lane roads situated in the south suburb of Paris (cf Fig. 1). Road $B-D$, connecting Paris to its south suburb, carries a high traffic volume, while road $A-C$ has a low traffic volume. Four incoming links and four inner zones at the centre of the junction are controlled by traffic lights. Special links are reserved for the right-turning vehicles.

The junction is fitted out with eight video cameras that capture four incoming and three outcoming links and four inner zones (cf Fig. 2). The location, height and angle of each camera depend on the geometry of the surveyed zone and are chosen to favour the measurement of space traffic parameters, for example, a queue length on incoming links. The camera views are analysed in real time, using image processing techniques developed at INRETS [12], in order to provide several measurements every second. The following traffic data are used for the OD matrix estimation:

- $\mathcal{X}_{i}(\tau)$ vehicle counts measured at the end of an entrance $i$ at second $\tau(i=1, \ldots, n)$,

- $\mathcal{Y}_{j}(\tau)$ number of vehicles that have passed through the beginning of the exit $j$ at second $\tau(j=1, \ldots, m)$,

- $\mathcal{Z}_{k}(\tau)$ number of stopped vehicles at inner zone $k$ at second $\tau(k=1, \ldots, q)$,

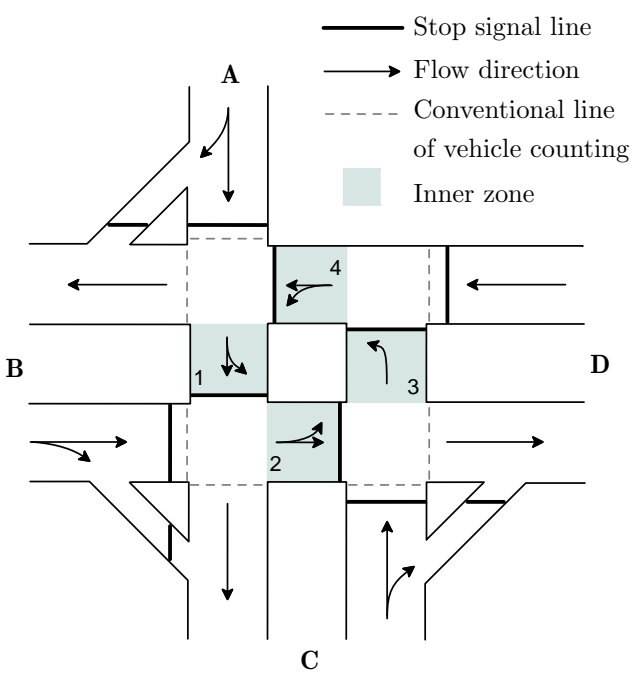

Fig. 1. The experimental junction

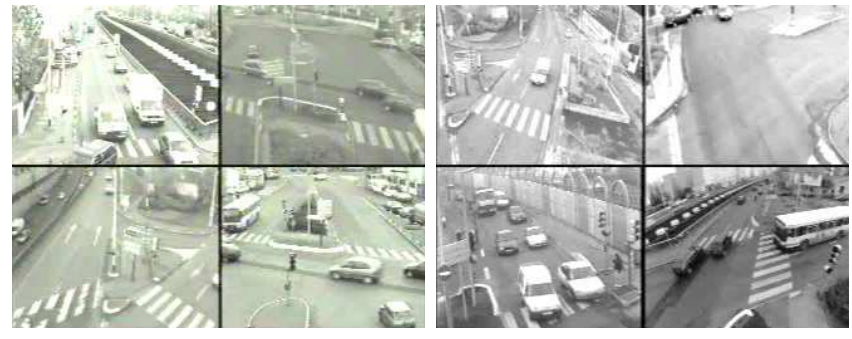

Fig. 2. Camera views

where $n, m$ and $p$ are the numbers of entrances, exits and inner zones respectively. Here, a traffic light cycle is a period of time between two sequential occurrences of the red light onset at one of the entries of road $B-D$.

It is worth noting that, generally, the measurements of traffic parameters are imprecise and erroneous. In particular, the vehicles counts are more imprecise than the measure of occupation rates of inner zones by stopped vehicles. Such a data imperfectness can be explained by different influencing factors. Thus, traffic count errors depend on traffic conditions. Count errors are greater during the peak periods when the gaps between vehicles are so small that it is difficult to distinguish these gaps on the video images.

The characteristics of vehicles are also a source of measurement errors. High vehicles passing in front of camera will hide the smaller vehicles or the whole camera field, i.e. they will produce a masking effect. Two-wheeled vehicles are only seldom counted because they are small.

Meteorological conditions inevitably influence all types of traffic measurements. Video images are blurred when the wind shakes the posts the cameras are fixed to, the sun's rays cause the reflections on the vehicle surfaces and camera lenses, and when rain, snow and fog obscure a camera field. Changes in brightness caused by the position of the sun, clouds and headlights at night also impact the quality of the measurements.

Let $x_{i}(c)$ be the flow volume at entrance $i$ during a traffic light cycle $c$ and $y_{j}(c)$ be the flow volume which entered the junction during cycle $c$ and leaves it by exit $j$. OD flow rate $b_{i j}(c)$ is the proportion of the flow of vehicles that come from entrance $i$ and go to exit $j$. The problem is to estimate OD flow rates $b_{i j}(c)(\forall i \in \llbracket 1, n \rrbracket, \forall j \in \llbracket 1, m \rrbracket)$ at the end of each traffic light cycle $c$, such that

$$
\begin{gathered}
y_{j}(c)=\sum_{i=1}^{n} b_{i j}(c) x_{i}(c), \\
x_{i}(c) b_{i j}(c) \geq z_{k i j}(c) \quad \forall k \text { s.t. } \delta_{k i j}=1, \\
\sum_{j=1}^{m} b_{i j}(c)=1 \\
b_{i j}(c) \geq 0
\end{gathered}
$$

where $z_{k i j}(c)$ is the number of vehicles which cross the junction from $i$ to $j$ and stop at inner zone $k$ during cycle $c, \delta_{k i j}=1$ if OD flow from $i$ to $j$ can pass through inner zone $k$ and is 0 otherwise. 
For a given cycle $c$ the value of variable $x_{i}(c)$ can be obtained from instantaneous vehicle counts

$$
x_{i}(c)=\sum_{\tau=1}^{\mathcal{G}_{i}(c)} \mathcal{X}_{i}(\tau),
$$

where $\mathcal{G}_{i}(c)$ is a duration of the green light of cycle $c$ in entrance $i$. However, a considerable number of vehicles, approaching to the junction with a high speed, enter the junction during some short period $\Delta \mathcal{R}_{i}$ at the beginning of the red light. Thus, to calculate a correct value of entrance flow volume $x_{i}(c)$, it is necessary to specify the duration of $\Delta \mathcal{R}_{i}(\forall i=\llbracket 1, n \rrbracket)$.

The values of $y_{j}(c)$ and $z_{k i j}(c)$ cannot be obtained directly from traffic measurements, because it is impossible to know exactly the period of time during which the vehicle flow $x_{i}(c)$ leaves the junction or stops at inner zones. Moreover, depending on the traffic control strategy and the flow volume, an OD flow may be divided on several platoons that don't leave the junction at the same period of time. For example, a flow coming from entrance $C$ to the exit $B$ is often separated in two platoons: the first one crosses the junction and doesn't stop at inner zones, whereas the second one has to stop at 4-th inner zone when its traffic light switches to red and will exit the junction later.

In order to obtain the values of $y_{j}(c)$ and $z_{k i j}(c)$ from $\mathcal{Y}_{j}(\tau)$ and $\mathcal{Z}_{k}(\tau)$ respectively, we propose to model a segmentation of OD flows on platoons via two Fuzzy-Timed High-Level Petri Nets. The first net provides the beginning and the end of each platoon composing an OD flow, whereas the second net gives the end of this OD flow. For a given cycle we thus know the possible duration of the presence of OD platoons at each junction zone and can collect the corresponding measurements during the same period. Therefore it is possible to put into a one-to-one correspondence the flows crossing the junction zones and the measurements taken at this zones. Consequently, a vehicle conservation law, represented by a consistent underdetermined system of equations whose unknowns are the OD flow rates, can be build dynamically and solved at each traffic light cycle.

\section{TRAFFIC FLOW SEGMENTATION USING FTHNS}

\section{A. Fundamentals of FTHNs}

Fuzzy-Timed High-Level Petri net (FTHN) is mathematical and graphical tool used to model discrete-event dynamic systems [13]. In difference of a Petri net [14] and a HighLevel (or Coloured) Petri net [15], [16], an FTHN considers a temporal evolution of a system state and temporal imprecision related to a system state change. Thus an FTHN is a Petri net endowed with notions of time and colour and with some concepts of fuzzy set theory. Its graphical representation is a directed graph composed of two types of nodes: places $p$, drawn by circles, and transitions $t$, represented by bars. A directional arc links solely a pair of nodes of different types. A system state is described by place marking $\mathcal{M}(p)$ which is a collection of coloured tokens with fuzzy timestamps. A

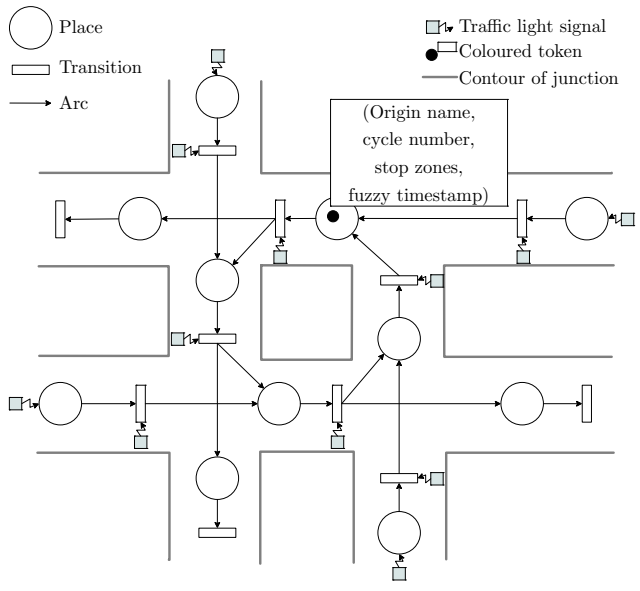

Fig. 3. The Fuzzy-Timed High-Level Petri Net for traffic flows crossing the signalised junction

change of this state, as well as a place marking change, can be involved when one or more events, represented by transitions, occur. Thereby, a dynamic behaviour of system is expressed by means of time-varying marking.

An 8-tuple $N=(\Sigma, \mathcal{P}, \mathcal{T}, \mathcal{A}, \mathcal{C}, \mathcal{W}, \mathcal{F}, \Pi)$ is considered in this paper as a formal representation of FTHN, where:

- $\Sigma$ is a finite non-empty colour set,

- $\mathcal{P}$ and $\mathcal{T}$ are the finite sets of places and transitions respectively, such that $\mathcal{P} \cap \mathcal{T}=\emptyset$ and $\mathcal{P} \cup \mathcal{T} \neq \emptyset$,

- $\mathcal{A} \subset(\mathcal{P} \times \mathcal{T}) \cup(\mathcal{T} \times \mathcal{P})$ is the finite set of arcs,

- $\mathcal{C}: \mathcal{P} \rightarrow 2^{\Sigma}$ is a colour function which associates a set of colours to a place,

- $\mathcal{W}: \mathcal{A} \rightarrow 2^{\Sigma}$ is an arc function,

- $\mathcal{F}$ is a delay associated to an arc,

- $\Pi$ is a set of fuzzy timestamps $\pi(\theta): \Theta \rightarrow[0,1]$, where $\Theta$ is a time scale.

A token is represented by a triple $(p, s, \pi(\theta))$, where $p \in \mathcal{P}$ is the place marked by the token, $s \subseteq \mathcal{C}(p)$ is a colour set which describes the system state in $p$, called colour, and $\pi(\theta)$ is the degree of possibility that the token arrives at the place and the system state changes at time $\theta$, called fuzzy timestamp. Marking $\mathcal{M}(p, \tau)$ of place $p \in \mathcal{P}$ at time $\tau$ is a multiset represented by the pair $(S, \mathbf{n})$ where $S$ is the set of colours of tokens arrived in $p$ at $\tau=\theta$ and $\mathbf{n}$ is the vector every element of which is the number of occurrences of the token's colour $s$ in set $S$.

Function $\mathcal{W}(p, t)$, corresponding to the arc from output place $p$ to $t$, is a multiset of colours that are elements of the set $\mathcal{C}(p)$. The function $\mathcal{W}(t, p)$ is defined similarly.

The set of input and output places of a transition is also interpreted as a set of pre- and post-conditions of an event $(\cdot p$ and $p \cdot$. Transition $t \in \mathcal{T}$ is enabled if $\mathcal{M}(\cdot p, \tau) \supseteq \mathcal{W}(\cdot p, t)$, i.e. precondition $\cdot p$ is fulfilled. If postcondition $p \cdot$ satisfies $\mathcal{M}(p \cdot, \tau) \supseteq \mathcal{W}(t, p \cdot)$, the enabled transition fires: it removes $\mathcal{W}(\cdot p, t)$ tokens from each of its input places $\cdot p$ of $t$ and adds $\mathcal{W}(t, p \cdot)$ tokens to its output places $p$. with delay $\mathcal{F}(t, p \cdot)$ associated to an arc from $t$ to $p \cdot$.

Note that many research works have contributed to a 
development of different frameworks of FTHN. For example, in [17] a marking of a place is a fuzzy set, in [18] a $T$-norm, associated to a transition $t$, represents a level of evidence that $t$ is enabled. A complete overview of fuzzy-timed Petri nets is proposed in [19].

\section{B. Structure of the FTHNs for vehicle flows crossing the junction}

In order to construct in a dynamical way a vehicle conservation law, we propose to build a model composed of two FTHNs. The topologies of these nets are the same and represent the junction structure, but the meanings of net elements and functions associated to them are different.

In the first net, a pair, place and transition, are associated to each zone of the experimental junction (Fig. 3). For entrances and inner zones, a transition represents a change of traffic lights, whereas for exits, it stands for the departure of a flow from the junction. A place indicates the presence of the flow at the corresponding junction zone by means of marking.

A token in place $p$ is represented by a triple $(p, s, \pi(\theta))$, where $s=\left(s_{1}, s_{2}, s_{3}\right)$ is a colour set containing the name of flow origin $s 1 \in\{A, B, C, D\}$, a cycle number $s 2 \in \mathbb{N}$ and a set of stop zones $s 3$, and $\pi(\theta) \in[0,1]$ is the degree of possibility that the token is present in place $p$ at time $\theta$, called fuzzy timestamp. Therefore, a token in place $p$ means the possible presence of a flow of origin $s 1$ in the zone corresponding to $p$. If $\pi(\theta)=1$, the flow is certainly present in a zone, if $\pi(\theta)=0$ the flow is absent. $\pi(\theta)$ takes value from interval $] 0,1[$ at the beginning of the red light when some vehicles could cross a stop line.

Two types of function are associated to an arc from transition $t$ to its output places $p \cdot$. First, weight function $\mathcal{W}(t, p \cdot)$ is defined over a set of colours $s 1$ representing the origins of the flows that can pass through this arc. Second, delay function $\mathcal{F}(t, p \cdot)$ is an average vehicle travel time between two corresponding successive zones of the junction.

The rules for changing colours of tokens, enabling and firing transitions and marking places are defined as follows.

1) Modelling of a traffic flow presence at the entries of the junction: During green light $\mathcal{G}_{i}(c)$ the traffic flow is certainly present at entrance $i$ and the corresponding place is marked by one token with colour $s=(s 1, s 2, s 3), s_{3}=\emptyset$, and with fuzzy timestamp $\pi(\tau)=1$, every second $\tau \in \mathcal{G}_{i}(c)$. Since the incoming flow may enter the junction at the beginning of the red light, $\Delta \mathcal{R}_{i}$, the place is marked by a token with timestamp $\pi(\tau)=1-\Delta \tau / \Delta \mathcal{R}_{i}$, where $\Delta \tau$ is a red light duration and $\Delta \mathcal{R}_{i}$ is a clearance time, equal to $2 \mathrm{sec}$. for the experimental junction.

In order to model the temporal imprecision when calculating entrance flow volume (2), we suggest to consider instantaneous vehicle counts $\mathcal{X}_{i}(\tau)$ at entrance $i$ as fuzzy sets $\tilde{\mathcal{X}}_{i}(\tau)=$ $\left\{\left(\mathcal{X}_{i}(\tau), \pi_{i}(\tau)\right), \mathcal{X}_{i}(\tau) \in \mathbb{R}\right\}$, where $\pi_{i}(\tau)$ is a membership function. A scalar cardinality of fuzzy set $\tilde{\mathcal{X}}_{i}(\tau)(\forall \tau \in c)$ is a total flow volume at entrance $i$ during cycle $c$ estimated as follows:

$$
x_{i}(c)=\sum_{\tau=1}^{\mathcal{G}_{i}(c)+\Delta \mathcal{R}_{i}} \mathcal{X}_{i}(\tau) \pi_{i}(\tau) \quad \forall i=\llbracket 1, n \rrbracket
$$

If the place, associated to entrance $i$, is marked, transition $t$, corresponding to the same zone, becomes enabled and fires by transmitting the token to the output places of $t$.

2) Modelling of traffic flow presence at the inner zones of the junction: The transition associated with inner zone $k$ is enabled if its input place $\cdot p$ contains at least one token during the green traffic light or the clearance time. The enabled transition fires, i.e. transmits the token downstream according to $\mathcal{W}(t, p)$ and $\mathcal{F}(t, p)$. When the traffic light is red, out of clearance period $\Delta \mathcal{R}_{k}$, tokens are stacked at place $\cdot p$ and the name of inner zone $k$ is added to colour set $s_{3}$ of each token in $k$. If the marking doesn't change during some period $\Delta \tau$, the value of $\max \left\{\mathcal{Z}_{k}(\tau)\right\}_{\forall \tau \in \Delta \tau}$, is associated to a set of flows possibly present in $k(5)$.

After the traffic light corresponding to zone $k$ switches again to green, the stopped vehicle platoon lefts this zone during period $\Delta \mathcal{G}_{k}$ proportional to volume of platoon. Thus the place, related to $k$, emits the stacked tokens endowed with fuzzy timestamps $\pi(\tau)=1-\Delta \tau / \Delta \mathcal{G}_{k}$, where $\Delta \tau$ is a green light duration and $\Delta \mathcal{G}_{k}$ is a period at the beginning of the green light when $\mathcal{Z}_{k}(\tau) \neq 0$. These tokens are shifted to the exit place $p$ with predefined delay $\mathcal{F}(t, p)$. According to hypothesis FIFO ("first in, first out"), none of upperstream flows can distance the platoon leaving inner zone $k$. It means that during $\Delta \mathcal{G}_{k}$ place $p$ emitting the staked tokens cannot be marked by upperstream tokens, i.e. its input transitions don't fire. Note that if $\mathcal{Z}_{k}(\tau)=0$ during period $\mathcal{R}_{k}(c)$ and the upperstream flows are not the same that the flows composing stopped platoon, time interval $\Delta \mathcal{G}_{k}$ is reduced to $1 \mathrm{sec}$.

3) Modelling of traffic flow presence at the exits of the junction: The transition related to the exit $j$ of junction is enabled and fires when each of its input places $p$ contains at least one token. When the marking of $p$ does not change during certain period of time $\Delta \tau$, each measure of exit flow volume over $\Delta \tau$ is bound to the set of entrance flows present at exit zone. A marking change of place indicates a change of a composition of flows present at $p$ and, for a given cycle $c$, segments each exit flow $y_{j}(c)$ into $L_{j}$ vehicle platoons, such that

$$
\begin{array}{cl}
y_{j}^{l}(c)=\sum_{\tau \in \Delta \tau_{l}(c)} \mathcal{Y}_{j}(\tau) & \forall l \in \llbracket 1, L_{j}(c) \rrbracket, \\
& \forall l \in \llbracket 1, L_{j}(c) \rrbracket, \\
z_{k i j}^{l}(c)=\max _{\tau \in \mathcal{R}_{k}(c)} \mathcal{Z}_{k}(\tau) \delta_{k i j} & \forall i \in \mathcal{M}\left(p, \Delta \tau_{l}\right), \\
& \forall k \text { s.t. } \delta_{k i j}=1,
\end{array}
$$

where $\Delta \tau_{l}(c)$ is a period of time during which platoon $l$ has left the junction, $z_{k i j}^{l}(c)$ is a maximum number of vehicles that belong to platoon $l$ and have stopped at inner zone $k, \mathcal{R}_{k}(c)$ is a red light duration in inner zone $k\left(\Delta \mathcal{R}_{k} \notin \mathcal{R}_{k}(c)\right), \delta_{k i j}$ indicates if zone $k$ is situated between OD pair of zones $(i, j)$, $\mathcal{M}\left(p, \Delta \tau_{l}\right)$ is a marking of place $p$ corresponding to exit $j$. 
The second FTHN, providing the ends of the flows, has the same meanings for places and transitions as the first FTHN. A token stands for the end of a flow and is represented by a set of two colours without timestamp: the name of flow origin $s 1 \in\{A, B, C, D\}$ and a cycle number $s 2 \in \mathbb{N}$.

The onsets of the beginning and the end of a flow in place $p$ allow us to determine the duration of the flow presence in zone related to $p$. Thus we can collect the measurements made in this zone and, at each cycle $c$, can built the following set of equations

$$
\begin{array}{cl}
y_{j}^{l}=\sum_{i \in \mathcal{M}\left(p_{j}, \Delta \tau_{l}\right)} x_{i} b_{i j}^{l} \pi_{i j}^{l} & \forall j \in \llbracket 1, m \rrbracket, \forall l \in \llbracket 1, L_{j} \rrbracket, \\
x_{i} b_{i j}^{l} \pi_{i j}^{l} \geq z_{k i j}^{l} & \forall i \in \mathcal{M}\left(p_{j}, \Delta \tau_{l}\right), \\
& \forall k \text { s.t. } \delta_{k i j}=1, \\
\sum_{j, l} \pi_{i j}^{l} b_{i j}^{l}=1 & \forall i \in \llbracket 1, n \rrbracket, \\
& \\
b_{i j}^{l} \geq 0 & \forall j \in \llbracket 1, m \rrbracket, \forall l \in \llbracket 1, L_{j} \rrbracket, \\
& i \in \mathcal{M}\left(p_{j}, \Delta \tau_{l}\right),
\end{array}
$$

where the cycle $c$ has been omitted to simplify the notations.

Let $J$ and $K$ be the numbers of constraints (6a) and (6b) respectively, and $I$ be the number of unknowns $b_{i j}^{l}$, such that

$$
\begin{array}{r}
J=\sum_{j=1}^{m} j L_{j}, \quad K=\sum_{j=1}^{m} \sum_{l=1}^{L_{j}} \sum_{i \in \mathcal{M}\left(p_{j}, \tau_{l}\right)} \sum_{k=1}^{q} \delta_{k i j}, \\
I=\sum_{j=1}^{m} \sum_{l=1}^{L_{j}} \sum_{i \in \mathcal{M}\left(p_{j}, \tau_{l}\right)} i .
\end{array}
$$

A matrix form of relationships $(6 \mathrm{a}-6 \mathrm{~d})$ can be written as :

$$
\begin{array}{r}
\mathbf{y}=\mathbf{X}_{\mathbf{1}} \mathbf{b} \boldsymbol{\pi}^{\top}, \\
\mathbf{X}_{\mathbf{2}} \mathbf{b} \boldsymbol{\pi}^{\top} \geq \mathbf{z}, \\
\mathbf{I b} \boldsymbol{\pi}^{\top}=\mathbf{1}, \\
\mathbf{b} \geq 0,
\end{array}
$$

where $\mathbf{y}$ and $\mathbf{z}$ are vectors composed of $J$ and $K$ elements respectively, $\mathbf{b}$ is a vector containing the $I$ unknowns $b_{i j}, \boldsymbol{\pi}$ is a vector composed of $I$ elements $\pi_{i j}^{l}, \mathbf{X}_{\mathbf{1}}$ is an $J \times I$ matrix rearranged so that (7a) is equal to (1a), $\mathbf{X}_{2}$ is an $K \times I$ matrix built so that (7b) is equivalent to (1b), $\mathbf{I}$ is an indicator $n \times I$ matrix organised in such a way that (7c) is equal to (1c) and $\mathbf{1}$ is an identity $n$-vector. Note that for the clarity of notation, the cycle $c$ has been omitted.

The number of unknowns $I$ depends on the junction structure and on the number of platoons that compose an OD flow. The number of equations $J$ depends on the number of exits and, in the same manner, the number of platoons. Since $I \geq J$ (in this paper $I \approx 15$ ), the system of equations ( $7 \mathrm{a})$ is underdetermined.

\section{OD FLOW RATES ESTIMATION}

Before estimating the OD flow rates from (7a) with respect to constraints $(7 b-7 d)$, it is necessary to consider two aspects of the problem : the system under-determination, appearing when $I>J$, and the system inconsistency that comes from the inequality $\sum_{i=1}^{n} x_{i}(c) \neq \sum_{j=1}^{m} y_{j}(c)$. In order to model the inherent data imprecision we suggest to consider the elements of the constraints (7a-7b) as triangular fuzzy numbers. The system under-determination can be overcame by applying the fuzzy linear programming problem as it is described below.

Let $\tilde{\mathbf{X}}_{\mathbf{1}}, \tilde{\mathbf{X}}_{\mathbf{2}}, \tilde{\mathbf{y}}, \tilde{\mathbf{z}}$ and $\tilde{\mathbf{b}}$ be triangular fuzzy numbers represented as triple $\tilde{\mathbf{a}}=\left(\mathbf{a}^{-}, \mathbf{a}^{\mathbf{m}}, \mathbf{a}^{+}\right)$. The form of fuzzy numbers $\tilde{\mathbf{X}}_{1}$ was inferred from empirical distributions of the error counts as shown in Fig. 4. The fuzzy number $\tilde{\mathbf{X}}_{2}$ is determined similarly to $\tilde{\mathbf{X}}_{1}$. In most cases, the counts of vehicles at the entrances are smaller than the true value of $\mathbf{X}_{1}$. Since there is no available histograms of error counts for $\mathbf{y}$ and $\mathbf{z}$, fuzzy numbers $\tilde{\mathbf{y}}$ and $\tilde{\mathbf{z}}$ are supposed to be symmetrical with the spreads chosen experimentally. One of the way for interpreting the equality of two fuzzy numbers $\tilde{a}$ and $\tilde{b}$ (cf [20]) is to consider ${ }^{\alpha} A \subseteq{ }^{\alpha} B(\forall \alpha \in[0,1])$, where the notation ${ }^{\alpha} A=\left\{x \mid \mu_{A}(x) \geq \alpha\right\}$ stands for the $\alpha$-cut of fuzzy number ã. In the same manner we define $\tilde{a} \geq \tilde{b}$ as a least conservative inequality $\sup \left({ }^{\alpha} A\right) \geq \inf \left({ }^{\alpha} B\right), \forall \alpha \in[0,1]$.

Following an interval regression approach proposed by Inuiguchi [21] and supposing the unknown OD flow rates $\tilde{\mathbf{b}}$ to be triangular fuzzy numbers of the form $\left(\mathbf{b}^{-}, \mathbf{b}^{m}, \mathbf{b}^{+}\right)$, we propose to estimate the following minimisation problem :

$$
\begin{array}{r}
\min _{\mathbf{b}^{m}, \mathbf{b}^{+}, \mathbf{b}^{-}, \mathbf{e}_{\mathbf{1}}, \mathbf{e}_{\mathbf{2}}, \mathbf{e}_{\mathbf{3}}}\left(\sum_{\alpha=1}^{N} \sum_{i=1}^{I}\left({ }^{\alpha} \mathbf{b}_{i}^{+}-{ }^{\alpha} \mathbf{b}_{i}^{-}\right)+\gamma_{1} \sum_{j} e_{1, j}+\right. \\
\left.+\gamma_{2} \sum_{j} e_{2, j}+\gamma_{3} \sum_{k} e_{3, k}\right)
\end{array}
$$

s.t.

$$
\left\{\begin{array}{l}
{ }^{\alpha} \mathbf{X}_{1}^{-\alpha} \mathbf{b}^{-} \boldsymbol{\pi}^{\top} \leq{ }^{\alpha} \mathbf{y}^{-}+\mathbf{e}_{\mathbf{1}}, \\
{ }^{\alpha} \mathbf{X}_{1}^{+\alpha} \mathbf{b}^{+} \boldsymbol{\pi}^{\top}+\mathbf{e}_{\mathbf{2}} \geq{ }^{\alpha} \mathbf{y}^{+}, \\
{ }^{\alpha} \mathbf{X}_{2}^{+\alpha} \mathbf{b}^{+} \boldsymbol{\pi}^{\top}+\mathbf{e}_{\mathbf{3}} \geq{ }^{\alpha} \mathbf{z}^{-}, \\
0 \leq{ }^{\alpha_{i}} \mathbf{b}^{-} \leq \mathbf{b}^{m} \leq{ }^{\alpha_{i}} \mathbf{b}^{+} \leq 1, \quad \forall i \in \llbracket 1, N \rrbracket \\
{ }^{\alpha} \mathbf{b}^{-} \leq{ }^{\alpha_{j}} \mathbf{b}^{-} \leq{ }^{\alpha_{j}} \mathbf{b}^{+} \leq{ }^{\alpha_{i}} \mathbf{b}^{+}, i<j, \forall i, j \in \llbracket 1, N \rrbracket \\
\mathbf{I b}^{m} \boldsymbol{\pi}^{\top}=\mathbf{1}, \\
\mathbf{e}_{\mathbf{1}} \geq 0, \mathbf{e}_{\mathbf{2}} \geq 0, \mathbf{e}_{\mathbf{3}} \geq 0,
\end{array}\right.
$$

where $N$ is a finite number of $\alpha$-cuts, $\gamma_{1}, \gamma_{2}$ and $\gamma_{3}$ are given weight coefficients, $\mathbf{e}_{\mathbf{1}}, \mathbf{e}_{\mathbf{2}}, \mathbf{e}_{\mathbf{3}}$ are the slack variables and the
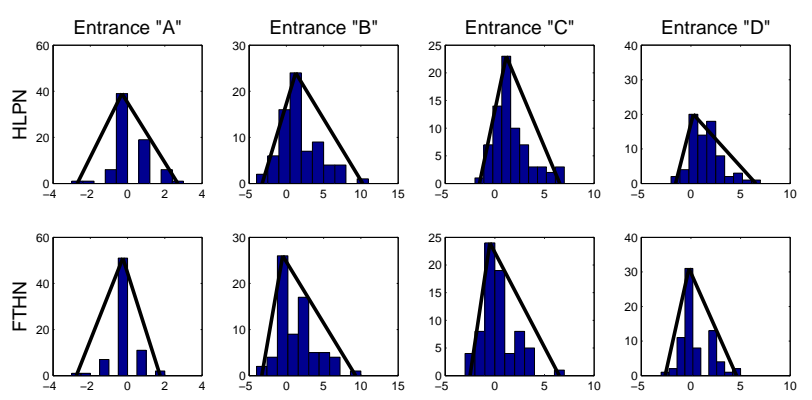

Fig. 4. Empirical distributions of error counts for the entrances of the junction 

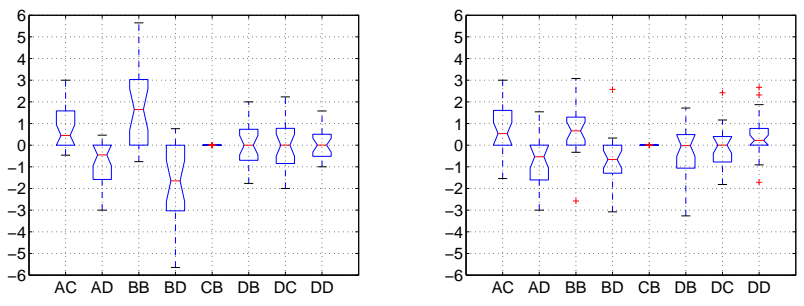

Fig. 5. Count errors (in vehicles) of FLP method using an HLPN (left) and an FTHN (right)

$\alpha$-cuts of fuzzy numbers $\tilde{\mathbf{X}}_{\mathbf{1}}, \tilde{\mathbf{X}}_{\mathbf{2}}, \tilde{\mathbf{y}}$ and $\tilde{\mathbf{z}}$ are calculated as follows:

$$
\begin{aligned}
& { }^{\alpha} \mathbf{X}_{1}^{-}=\alpha\left(\mathbf{X}_{1}^{m}-\mathbf{X}_{1}^{-}\right)+\mathbf{X}_{1}^{-}, \quad{ }^{\alpha} \mathbf{X}_{1}^{+}=\alpha\left(\mathbf{X}_{1}^{m}-\mathbf{X}_{1}^{+}\right)+\mathbf{X}_{1}^{+}, \\
& { }^{\alpha} \mathbf{X}_{2}^{+}=\alpha\left(\mathbf{X}_{2}^{m}-\mathbf{X}_{2}^{+}\right)+\mathbf{X}_{2}^{+}, \quad{ }^{\alpha} \mathbf{y}^{-}=\alpha\left(\mathbf{y}^{m}-\mathbf{y}^{-}\right)+\mathbf{y}^{-}, \\
& { }^{\alpha} \mathbf{y}^{+}=\alpha\left(\mathbf{y}^{m}-\mathbf{y}^{+}\right)+\mathbf{y}^{+}, \quad{ }^{\alpha} \mathbf{z}^{-}=\alpha\left(\mathbf{z}^{m}-\mathbf{z}^{-}\right)+\mathbf{z}^{-} .
\end{aligned}
$$

Note that the fifth constraints of (9) shows that $\left[{ }^{\alpha_{i}} \mathbf{b}^{-},{ }^{\alpha_{i}} \mathbf{b}^{+}\right] \subseteq$ $\left[{ }^{\alpha_{j}} \mathbf{b}^{-},{ }^{\alpha_{j}} \mathbf{b}^{+}\right]$, where $i<j, \forall i, j \in \llbracket 1, N \rrbracket$.

The method have been tested using real data collected at the experimental junction during 25 consecutive traffic light cycles $(\approx 30$ minutes) with peak traffic conditions. The actual values of OD flow rates $\boldsymbol{\beta}=\left\{\beta_{i}\right\}_{i=1, \ldots, 8}$, calculated manually from video images, are available for all cycles. The tests are made using two nets HLPN and FTHN, where the HLPN is a configuration of FTHN with $\Delta \mathcal{R}_{i}=0, \forall i \in \llbracket 1,8 \rrbracket$, and $\Delta \mathcal{G}_{k}=0, \forall k \in \llbracket 1,4 \rrbracket$.

The support width of fuzzy numbers $\tilde{\mathbf{y}}$ and $\tilde{\mathbf{z}}$ were experimentally fixed to 2 . The weight coefficients has been fixed as follows: $\gamma_{1}=0.1, \gamma_{2}=0.1, \gamma_{3}=0.2$.

The estimation error has been calculated for the OD flow rates (Fig. 5): $E=\hat{\mathbf{b}} \mathbf{X}^{*}-\boldsymbol{\beta} \mathbf{X}^{*}$, where $\mathbf{X}^{*}$ is a vector of actual vehicle counts at the entrances of the experimental junction, $\hat{\mathbf{b}}$ is a vector of estimated OD flow rates. A significant gain is obtained when the temporal imprecision is modelled. Thus the best results are achieved using an FTHN for which the range of $E$ and the absolute values of three quartiles of $E$ are lower for most of OD flows. Whatever the configuration of Petri net, the estimation error $E$ is higher when the flow volume is lower, like for the OD flows "AD" and "BB".

\section{CONCLUSION}

A new short-time estimation method of the OD matrix for a signalised junction have been proposed in this paper. The method is founded on the construction of a conservation law of vehicles at each traffic light cycle, represented by the underdetermined system of equations. This system is obtained in a dynamical way from the model of traffic flows built using two Fuzzy-Timed High-Level Petri Nets (FTHN). Real data collected at the experimental signalised junction fitted out with video cameras are used to estimate the OD flow rates. FTHNs model a temporal imprecision of the data.

Fuzzy linear programming approach have been proposed to estimate the OD matrix. The inherent imprecision of the data was modelled by representing the data by triangular fuzzy numbers. The tests are made for two different configuration of FTHN such that one takes and second does not take into account the temporal imprecision of data. The best results were obtained in the second case.

Our future lines of research will be centred on the improvement of the FLP method in order to provide the best and unique estimation of the OD flow rates. The application of our method should be also extended to a sequence of junctions.

\section{REFERENCES}

[1] H. J. Van Zuylen, "The estimation of turning flows on a junction," Traffic Engineering Control, vol. 20, no. 11, pp. 539-541, 1979.

[2] E. Hauer et al., "Estimation of turning flows from automatic counts," Transportation Research Record, no. 795, pp. 1-7, 1981.

[3] M. J. Maher, "Inferences on trip matrices from observations on link volumes: a Bayesian statistical approach," Transportation Research Part $B$, vol. 17 , no. 6 , pp. $435-447,1983$.

[4] M. Cremer and H. Keller, "A new class of dynamic methods for the identification of origin-destination flows," Transportation Research Part $B$, vol. 21, no. 2, pp. 117-132, 1987.

[5] N. L. Nihan and G. A. Davis, "Recursive estimation of origin-destination matrices from input/output counts," Transportation Research Part B, vol. 21, no. 2, pp. 149-163, 1987.

[6] B. Li and B. De Moor, "Recursive estimation based on the equalityconstrained optimization for intersection origin-destination matrices," Transportation Research Part B, vol. 33, no. 3, pp. 203-214, 1999.

[7] H. D. Sherali et al., "Parameter optimization methods for estimating dynamic origin-destination trip-tables," Transportation Research Part B, vol. 31, no. 2, pp. 141-157, 1997.

[8] S. Midenet et al., "Signalized intersection with real-time adaptive control: on-field assessment of $\mathrm{CO}_{2}$ and pollutant emission reduction," Transportation Research Part D, vol. 9, pp. 29-47, 2004.

[9] H. Keller and G. Ploss, "Real-time identification of O-D network flows from counts for urban traffic control," in Proceedings of the 10th International Symposium on Transportation and Traffic Theory, N.H.Gartner and N.H.M.Wilson, Eds. Elsevier Science Publishing Co., 1987, pp. 267-289.

[10] I. Matschke and B. Friedrich, "Dynamic OD estimation using additional information from traffic signal lights timing," Proceedings of the Triennial Symposium on Transportation Analysis TRISTAN IV, 2001.

[11] K. Biletska, S. Midenet, M. Masson, and T. Denoeux, "Fuzzy modelling of the sensor data for the estimation of an origin-destination matrix," 2009, to appear in Proceedings of IFSA-EUSFLAT'2009, 20-24 July, Lisbon, Portugal.

[12] D. Aubert et al., "Automatic vehicle queue measurement at intersections using image-processing," 8th IEE International Conference on Road Traffic Monitoring and Control, London, vol. 422, pp. 100-104, 1996.

[13] T. Murata, T. Suzuki, and S. Shatz, "Fuzzy-timing high-level Petri nets (FTHNs) for time-critical systems," in Fuzziness in Petri Nets, in: Studies in Fuzziness and Soft Computing. Physica-Verlag, 1999, pp. 88-114.

[14] T. Murata, "Petri nets: properties, analysis and applications," in Proceedings of the IEEE, vol. 77, April 1989, pp. 541-580.

[15] K. Jensen, L. Kristensen, and L. Wells, "Coloured Petri nets and CPN tools for modelling and validation of concurrent systems," in International Journal on Software Tools for Technology Transfer. Springer Verlag, 1998, pp. 98-132.

[16] M. Dotoli and M. P. Fanti, "An urban traffic network model via coloured timed Petri nets," Control Engineering Practice, vol. 14, pp. 1213 - 1229, 2006.

[17] Z. Dinga, H. Bunkeb, O. Kipersztokc, M. Schneiderd, and A. Kandela, "Fuzzy timed Petri nets - analysis and implementation," Mathematical and Computer Modelling, vol. 43, pp. 385-400, 2006.

[18] W. Pedrycz and H. Camargo, "Fuzzy timed Petri nets," Fuzzy Sets and Systems, vol. 140, pp. 301-330, 2003.

[19] J. Cardoso and H. Camargo, Eds., Fuzziness in Petri Nets, ser. Studies in Fuzziness and Soft Computing. Physica-Verlag, 1999.

[20] D. Dubois and H. Prade, Eds., Fuzzy sets and Systems: Theory and Applications. Academic press, Inc., 1980.

[21] R. Slowinski, Ed., Fuzzy sets in decision analysis, operation research and statistics, ser. The handbooks of fuzzy sets. Kluwer Academic Publishers, 1998. 